Research Article

\title{
Efficiency and Safety in Operating Rooms: Current Limitations and Future Directions
}

\author{
Georgios-loannis Tsianos' ${ }^{1}$ Benedicte Dahlerup $^{2}$, Agnieszka Golińska ${ }^{3}$, Efstathios Vassiliadis ${ }^{4}$ \\ ${ }^{1,2,4}{ }^{2}$ ARK, ${ }^{3}$ EVNIA, Copenhagen Business Center, Hellerup Strandvejen 60, 2900, Copenhagen, Denmark. \\ DOI: https://doi.org/10.24321/2394.6539.201901
}

I $\quad \mathbf{N} \quad \mathbf{F} \quad \mathbf{O}$

\author{
Corresponding Author: \\ Georgios-loannis Tsianos, Copenhagen Business \\ Center, Hellerup Strandvejen 60, 2900, \\ Copenhagen, Denmark. \\ E-mail Id: \\ tgi@oark.dk \\ Orcid Id: \\ https://orcid.org/0000-0001-9501-0903 \\ How to cite this article: \\ Tsianos GI, Dahlerup B, Golińska A et al. Efficiency \\ and Safety in Operating Rooms: Current \\ Limitations and Future Directions. J Adv Res \\ Med Sci Tech 2019; 6(1\&2): 1-6. \\ Date of Submission: 2019-08-03 \\ Date of Acceptance: 2019-09-07
}

\section{$\begin{array}{llllllll}\mathbf{A} & \mathbf{B} & \mathbf{S} & \mathbf{T} & \mathbf{R} & \mathbf{A} & \mathbf{C} & \mathbf{T}\end{array}$}

Studies on clinical adverse events in hospitals have identified the Operating Room (OR) as the most probable place for medical error. Therefore, it is highly important to improve efficiency and safety within the OR. We decided to review the current available evidence in the literature on the topic of OR efficiency and safety; we identified the limitations and pitfalls within the OR, searched for the roots for their existence and described future directions and recommendations. In addition, we explored the economic cost implications as they pertain to the OR use under those limitations, all of which synthetically, bring forward the necessity that things need to change within the OR otherwise a healthcare unit's finances will continue to take significant losses. Finally, we briefly describe a future solution for the OR.

Keywords: Operating Rooms, Operating Room Economics, Operating Room Ergonomics, Patient Safety

\section{Introduction}

The Operating Room (OR) is the central hub of any hospital, and therefore maximising OR efficiency in the way that it is managed and operated, this will ultimately have important implications on patient safety, patient satisfaction, medical team morale and overall hospital cost savings. On the other hand, operating room safety is directly related to patient safety in surgery and that remains a daily challenge in the OR for the OR personnel. In this review article, we will explore both OR safety and efficiency in order to identify the current limitations in an attempt to suggest solutions for the future.

Recent studies on clinical adverse events in hospitals have identified the Operating Room (OR) as the most probable place for medical error. ${ }^{1}$ All the patient and treatment protocols, the high level of technology used for surgeries that is constantly changing and evolving, as well as the fact that human life is at high risk, collectively make the OR the most complex, and expensive workplace in healthcare units. Thus, it is imperative to improve efficiency and safety within the area where lives are essentially being saved. The first step in achieving this includes identifying the limitations and their impact in the OR, then to specify the root of the limitations and finally to describe future solutions and recommendations that should be pursued and adapted in order to enhance the functionality of todays ORs. Finally, given the fact that most surgical cash are spent in the OR. ${ }^{2}$ The financial costs implicated in a sub-optimally utilized OR are also important as they ultimately dictate what can and cannot be achieved within an OR and the healthcare unit as a whole.

\section{Limitations and Pitfalls of the Operating Room}

The OR is a system constantly in flux, and amongst its major limitations are the delays and erroneous workflow. Cardoen B et al. ${ }^{3}$ reviewed the multiple assessments on OR planning and scheduling as means of having an efficient and effective 
running operating unit. Various criteria were shown to be linked with the OR's poor performance, from waiting time of patients and surgeons, utilization of OR resources i.e. over- or under-utilization, throughput of patients, ward/ICU beds, cancellations/deferrals of cases, and finally turn over time (TOT). ${ }^{3}$ Girotto JA et al. ${ }^{4}$ analysed a patient's journey from the surgeon's office, throughout the day of the surgery and until discharge. They observed that only $16 \%$ of the operations began within 5 minutes of their scheduled start. The group also noticed that a total non-operative room time was about 51 min, which was much more than the one documented in the hospital's ESI (electronically stored information), at $34 \mathrm{~min}$. An increased TOT can result from lack of equipment availability, unavailability of enough staff to turn over the room, or readiness of the next patient. ${ }^{5}$ Meredith JO et al. ${ }^{6}$ described three critical phases in TOT: (1) patient removal, (2) patient transition, and (3) operation preparation. Improvement of TOT depends on how efficient each of these phases can be. Time with no occurring surgical activity, also known as 'white space', contributes to inefficiency and time wasted. Examples of white space include instrument preparation, the positioning of the patient, as well as waiting for surgical staff or the patient. ${ }^{6}$ Weinbroum AA et al. studied the causes of the time wasted in the OR. The experiment lasted 30 days and the group counted 79 hours wasted (almost three quarters) of an OR's full working capacity each week. They identified 5 different areas where time was wasted:

- Surgeon unavailability. Total wasted time was 5.9 hours. It resulted from the surgeon's late arrival to the OR, or because a junior surgeon who needed help had to wait for a senior surgeon.

- $\quad$ Inappropriate patient preparation. It accounted for 9.6 hours of wasted time and appeared due to inadequate paperwork, missing laboratory results, unmarked operative site, improperly signed forms or obstacles encountered before surgery that required additional tests.

- Unavailable room or staff. 46.2 wasted hours due to OR occupied by emergency cases, and lack of medical staff.

- Cleaning time. $10 \%$ of the unused time was due to OR cleaning.

- Post-Anaesthesia Care Unit (PACU) space and transport. 7.75 hours were wasted due to PACU congestion, while a total of 1.95 hours due to unavailable transport to PACU.

A lot of OR time is unused during induction of anaesthesia, waiting for a place in PACU or waiting for available transport to it. The root of this problem is that all pre and postoperative action takes place within the OR. As a result, delays accumulate, and the OR instead of being an area for surgeries is used as a waiting room. ${ }^{8}$ Another cause for the delays is the long preparation of surgical instruments.
This is one of the most complex and time-consuming processes during the TOT, and it is often the case that instrument trays are stacked due to lack of space to lay them out. As a result, a scrub nurse, in order to locate the proper tool, has to make unnecessary movements and thus ultimately waste time. ${ }^{6}$ The rest of the delays are mainly due to an insufficient number of nurses and surgeons, or poorly trained medical staff, as well as the lack of communication between them. A potential cause of the wrong communication between OR staff includes poor timing, inaccurate information, unresolved issues or the fact that the leaders are not involved in the exchange of the most important information. ${ }^{9}$

Looking at the issues more closely within the area the OR, and more specifically the actual working environment. Matern $U$ et al. ${ }^{10}$ studied the working conditions in the OR and carried out a survey among surgeons working in hospitals. The obtained results showed how poorly ORs are equipped and managed. When asked about the ambient temperature, only $31 \%$ of the participants answered that it is suitable. Most of the surgeons (84\%) stated that their working posture was painful and uncomfortable. In addition, $67 \%$ of the surgeons considered the operating elements of the OR tables to be inadequate as they had a lot of difficulties in obtaining the correct position of the patients, $35 \%$ said that an optimal placement of the monitor that displays the operation is not possible, while $71 \%$ experienced problems with using the OR lights. Matern $\mathrm{U}$ et al. ${ }^{10}$ also discovered that $93 \%$ of the equipment used were separate units, whereas the integrated ones were not reliable. Moreover, while only $30 \%$ of the participating surgeons did not experience problems with the device control systems, the rest considered them as not intuitive. Furthermore, in most of the cases, equipment cables and tubes were placed in a way that hindered the work of the surgeons, thus posing a risk to both staff and ultimately patients. Last but not least, 59\% stated that they were not well trained in handling the equipment they were using. Badly designed and managed ORs can lead to serious consequences; an uncomfortable workplace environment with poorly adjusted and difficult to handle equipment, collectively foster mistakes and jeopardize the lives of patients. Berguer $\mathrm{R}^{11}$ gives an example of wrongly adjusted monitors causing neck stiffness and pain for the surgeons resulting in low level of performance and high level of dissatisfaction.

A badly designed working area, as studied by Matern $U$ et al. ${ }^{10}$ can result from the limited use of human factors design. Human factors are defined as "the application of the scientific knowledge of human capabilities and limitations to the design of systems and equipment to produce products with the most efficient, safe, effective, and reliable operation". ${ }^{12}$ In such a design, the interaction 
between equipment and the people who use them is taken into account and the machines therefore, or the OR, fit the human body and its cognitive abilities. Wrongly adjusted monitors, lights, or operating table will not occur anymore. In addition, the equipment is intuitive thus surgeons will not experience problems when using them, while further, cables and tubes are positioned in such a manner that don't get in the way of the OR staff. As a result, the operation will be faster and safer. It thus becomes clear how important OR ergonomics is Beurguer ${ }^{11}$ discusses the significant role of ergonomics in medicine whereby the design of machines and equipment in order to optimize performance by the user is directly related to patient safety. The Food and Drug Administration (FDA) has recognized the impact of ergonomics on patient safety, suggesting that up to half of the 1.3 million unintentional patient injuries in the United States hospitals each year may be a result of poorly designed medical instruments. ${ }^{13}$

To make matters more substandard, distractors, such as noise, influence a surgeon's concentration as well as communication between medical members, all leading to human stress and tension development in the OR. As a consequence, this translates to prolonged operation time, dissatisfaction of the personnel and high error rate. That error can include wrong site injury (i.e. surgery on the wrong patient, on the wrong organ, on the wrong limb, or on the wrong level), retained foreign body or injury of the personnel. Estimates of retained foreign bodies during operations range from 1 in 8,000 to 13,000 surgical operations, and up to 1 in every 5,000 abdominal operations, while wrong side injury occurs in approximately 1 in 113,000 operations. ${ }^{14}$ Bharathan $\mathrm{R}$ et al. ${ }^{15}$ state that $10 \%$ of patients admitted to hospitals suffer an adverse event, while in addition, $50 \%$ of all medical errors occur during surgeries, and about $40 \%$ of those within the OR. Arora $\mathrm{S}$ et al. ${ }^{1}$ investigated different factors compromising safety during surgery. They identified strong correlations between potentially stressful events and actual experienced stress. Technical problems, equipment related situations (such as lack of an instrument), as well as patient factors, were found to be the most frequent and stressful situations. Distractions (i.e. ringing phone) was another issue that appeared often, however, they didn't cause much stress. ${ }^{1}$ Finally, Kurmann A et al. ${ }^{9}$ discuss communications to be fundamental for patient safety and successful operation completion. According to them, communications and team breakdown are responsible for $38 \%$ of incidents in nonemergency situations and may impact even up to $59 \%$ of emergency incidents.

\section{Future Directions and Recommendation}

Based on the description of todays OR, it is clear that improvements are absolutely necessary. In order to increase the effectiveness and safety of an OR, distractors, interruptions and situations causing fatigue and other sources of stress have to be minimized, while communication and teamwork need to be maximized. Bharathan $\mathrm{R}$ et al. ${ }^{15}$ proposed an integrated OR as a solution to that problem. They stated that such an OR encompasses the use of communication protocols which are an important element in increasing the level of effectiveness of an OR. The group stated that case-irrelevant communication is one of the sources of distraction; redundant communication content can be removed, and a relevant communication protocol synchronized with individual tasks of the procedure be put in place by following the concept of a 'sterile cockpit'. Klein $\mathrm{M}$ et al. ${ }^{16}$ compared the efficiency of a standard OR with the Integrated OR (IOR), and noticed a significant decrease in physical complaints during operations whilst in the IOR. Another experiment showed that in 45 procedures analysed, the number of risk-sensitive events (RSE) in an IOR using an electronic checklist, the proportion of procedures with one or more RSE decreased anywhere from $7 \%$ to $47 \%$. Kenyon TAG et al. ${ }^{17}$ on the other hand, completed a simulation study of a dedicated IOR and observed a significant reduction of time spend on the 'setup' and 'put-away' of the equipment. Finally, preparation of the patient and sterility in the IOR is thought to be improved compared to the traditional one $\mathrm{e}^{15}$

Garbey $\mathrm{M}$ et al. ${ }^{18}$ proposed a real-time OR management platform as a tool for increasing safety and efficiency. They stated that their system is able to automatically track and record OR activity and thus warn appropriate OR staff if any events or their length jeopardize the life of a patient in real-time. Tadeo $\mathrm{JR}^{19}$ tested the implementation of a preoperative huddle $15 \mathrm{~min}$ before the first surgery start time for all elective surgical procedures; such practice fosters teamwork by defining the roles and duties of each member. In addition, discussing critical information about both patient and surgery, before and after the procedures, increases communication among the staff. ${ }^{20-21}$ leading to avoidance of perioperative team errors ultimately leading to safer procedures. Finally, a preoperative huddle improves patient satisfaction since they can witness the medical staff's dedication to carry out the surgery as safely and efficiently as possible. ${ }^{19}$

Improvement of the OR efficiency can also be achieved by lowering the TOT. TOT in the OR arises from multidimensional interactions and include additional factors such as staff arriving on time, cleaning procedures, surgical equipment preparation, time of day of operation. ${ }^{22-23}$ Meredith JO et al. ${ }^{6}$ noticed that when the surgeon arrives in the operating theatre before the patient, delays decrease and the team is motivated, while in addition, the surgeon has time to assist with minor preparations and jobs other than the surgery itself. Another proposition in the attempt to shorten patient preparation time has to do with the time lost in the OR during induction of anaesthesia. Therefore, a suggested 
solution would be to redesign the OR. One of the first such redesigns took place at the Massachusetts General Hospital. With that in mind, Sandberg WS et al. tested a three-room suite that included the OR, an induction room, and early recovery area [8] In such a type of construction, traditional sequential activities are run in parallel, and as a result, the new OR processed more cases per day and used less time per case..$^{8,24-26}$ Finally, of great value is the choice of the most appropriate team members in order to promote high performance as that pertains to OR efficiency through turnaround times. In the majority of the cases, a surgeon is placed to lead a surgical list; allocation of the right anaesthesiologist however to the list, and the surgeon, can positively affect the OR efficiency outcome. ${ }^{27}$ Successful teaming for OR efficiency extends to surgeons working with the team of nurses as well due to nurses' familiarity with the equipment, phraseology, awareness of surgeon's preferences and anticipation of their needs. ${ }^{28-29}$

Another way to improve todays OR is to work on nontechnical skills. First of all, special training of medical staff can help to minimize distractors. Surgeons should learn different strategies to introduce moments that require special attention or silence (i.e. developing formal rules). Another useful skill for surgeons is to be able to ignore a portion of distraction and focus on the specific task, and this can be achieved through surgical training under noisy conditions. Furthermore, special training can also influence the efficiency of the rest of the medical team.
Studies showed that the combination of training and introduction of a checklist caused fewer complications, a lower mortality rate and overall improved patient safety on a global scale. ${ }^{30}$ Such training should include exercises, case studies, and simulations. ${ }^{9}$ Finally, all team members ought to regularly work on improving patient preparation, as a significant amount of time is wasted due to inadequate paperwork, missing laboratory results, unmarked operative site, improperly signed forms or similar mistakes and all of these can be easily corrected.

Finally, Kopelman $Y$ et al. ${ }^{31}$ elaborated on the trends of the evolving technologies in the OR of the future. They discuss that in order to reduce the cost and increase the efficiency and safety of care, it is necessary to improve on the integration of high technology, communication, teamwork, as well as coordination between services, providers and staff. Furthermore, they mention that in the future OR, improvements of real-time workflow process should be implemented; this will include parallel processing, novel information technology architecture, as well as management and patient tracking solutions. In addition, thanks to future hybrid ORs, combined open, minimally invasive, image-guided and/ or catheter procedures will be carried out in the same OR and within the same operative setting. Advanced and complicated surgeries, however, will need different imaging systems, complex surgical table or a conveyor that moves patients between stations.

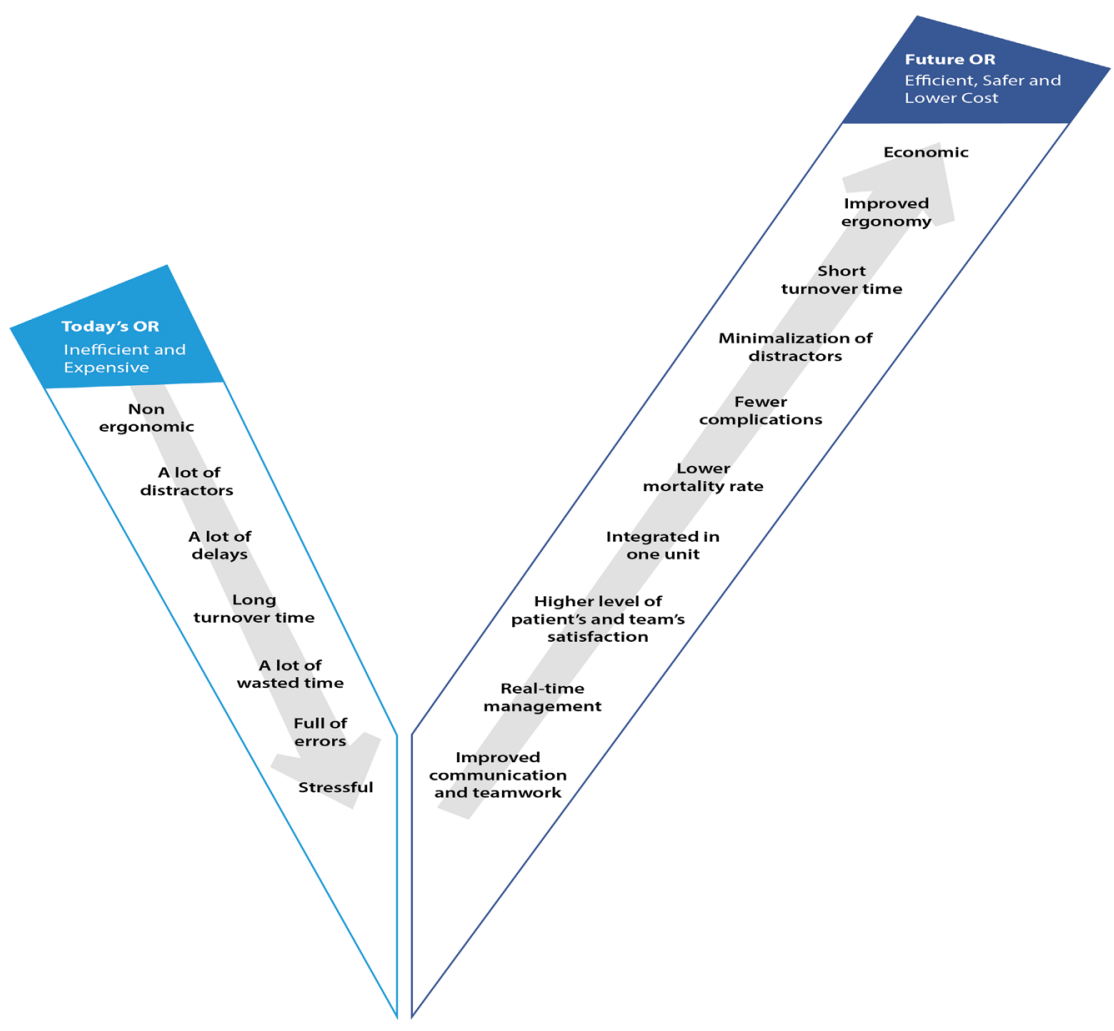

Figure I.Future OR solutions in order to create a safer and a more effective and efficient working and operating unit 


\section{Operating Room Economics}

OR management and running, as it's expected, does not only affect the OR's microenvironment, but has a colossal implication on a hospital's economy of scale. Hospitals, on one hand, want to maximize the level of patient safety and satisfaction, while on the other, would like to reduce costs and improve their financial assets. The hospital management teams are constantly struggling to improve the efficiency of OR use and it is becoming a major issue in their pursuit to provide high-quality health care. Regarding the financial aspect, the area that is of particular interest is the OR as it is the hospital's largest cost and revenue center. ${ }^{32-33}$ It becomes evident that as the surgical service is an expensive one to operate, ${ }^{34-35}$ it has a major impact on the performance of the hospital as a whole. It is thus important that the OR utilization rate remains high without having to increase resources in order to do so. OR utilization is directly affected by the scheduling and planning of surgical lists, starting of surgical lists on-time, and TOT. ${ }^{5}$ It is therefore not surprising that $O R$ efficiency and utilization decreases with an abnormal number of surgical cases scheduled, in excess or lack thereof, delays of surgical case start time, and of course, a prolonged TOT between cases. So why are OR economics so important and what does that actually mean in financial terms? This question is very important in business; if something cannot be measured effectively it cannot be managed effectively. ${ }^{36} \mathrm{At}$ a cost of even up to approximately $\$ 100 /$ minute at times, OR time is incredibly expensive and even small delays compounded over the course of multiple ORs and multiple days, can result in a significant impact. ${ }^{18}$ According to the Chief Financial Officer at the Southern California County Hospital (SCCH), the total cost per minute rate of OR operations for the year 2012-2013 was $\$ 51.86$; this figure accounted for all staff salaries, equipment and supplies. ${ }^{21}$ In addition, during a study of 100 hospitals, the average operating cost for an OR was $\$ 62.19$ per minute, with a range of responses from $\$ 22.80$ to $\$ 133.12$ per minute. ${ }^{37}$ while further, by including the average fee for the anesthesiologist, the average figure climbs to an average of $\$ 66 /$ minute in operating costs. Adjusting these values to account for inflation, the average operating costs for 2013 is $\$ 79.92$, with a range from $\$ 27.63$ to $\$ 156.81 .{ }^{37}$ Moreover, Girotto et al. (2010) designed a series of studies and analysed OR data from the University of Rochester Medical Centre (URMC), a large hospital system servicing the tertiary care needs of the New York Upstate. The URMC Office of Director quotes a cost of even up to $\$ 100 /$ minute, or $\$ 6,000 /$ hour, of unused operative time per OR, excluding physician time lost. In addition, it was estimated that the total delay time for "first start" patient cases ("first start" patients are those scheduled to start the day as the "first" patient in a given OR) approximates to about 98,100 min every year and costs up to $\$ 9.6$ million.
It becomes evident therefore, that any unused or wasted OR time is costing the health care units a significant share of their funds and over the course of a year, the amounts can reach well into the millions (depended of course on hospital size and ORs within it). ${ }^{4}$

\section{Future Operating Room Solution}

Given the presented limitations within the current OR setting and how these are seriously affecting both patient safety and staff wellbeing, in addition to the financial minefields that ORs are turning into, a disruptive and innovative OR solution is clearly needed. This is not a new concept and the need has been previously described as well; ${ }^{38}$ yet, such breakthrough innovation has yet to be brought forward. The solution should be expected to have advantages that are both qualitative and quantitative (Figure 1). The qualitative advantages will need to address safety issues from the elimination of the cable chaos, enhanced user-friendliness and satisfaction, improved HumanMachine Interface (HMI), integration of technologies, improved ergonomics, as well as the elimination of the single surgical specialty dedicated ORs. With regards to a major quantitative advantage, the future solution will be expected to significantly contribute towards the reduction of Turnover Time (TOT). This reduction in TOT will result in a reduced time to do tasks within the $O R$, while indirectly impact positively other areas outside the OR, all ultimately leading towards greater patient safety and massive cost savings for the hospital.

\section{Conflict of Interest: None}

\section{References}

1. Arora $\mathrm{S}$, Hull $\mathrm{L}$, Sevdalis $\mathrm{N}$ et al. Factors compromising safety in surgery: stressful events in the operating room. Am J Surg 2010; 199(1): 60-65.

2. Barbagallo S, Corradi L, de Ville de Goyet J et al. Optimization and planning of operating theatre activities: an original definition of pathways and process modeling. BMC Med Inform Decis Mak 2015; 15(1): 38.

3. Cardoen B, Demeulemeester E, Beliën J. Operating room planning and scheduling: A literature review. Eur J Oper Res 2009; 201: 921-932.

4. Girotto JA, Koltz PF, Drugas G. Optimizing your operating room: Or, why large, traditional hospitals don't work. Int J Surg 2010; 8(5): 359-367.

5. Li D, Wang S, Powers C. A systematic strategy for perioperative process improvement. In: IIE Annual Conference and Expo. 2013; 626-634.

6. Meredith JO, Grove AL, Walley P et al. Are we operating effectively? A lean analysis of operating theatre changeovers. Oper Manag Res 2011; 4(3-4): 89-98.

7. Weinbroum AA, Ekstein P, Ezri T. Efficiency of the operating room suite. Am J Surg 2003; 185(3): 244-250. 
8. Sandberg WS, Daily BA, Egan M et al. deliberate perioperative systems design improves operating room throughput. Anesthesiology 2005; 103(2): 406-418.

9. Kurmann A, Tschan F, Semmer NK et al. Human factors in the operating room - The surgeon's view. Trends Anaesth Crit Care 2012; 2(5): 224-227.

10. Matern U, Koneczny S. Safety, hazards and ergonomics in the operating room. Surg Endosc 2007; 21(11): 1965-1969.

11. Berguer R. Surgery and ergonomics. Arch Surg 1999; 134(9): 1011-1016.

12. King PH, Fries RC, Johnson AT. Design of biomedical devices and systems. CRC Press, Taylor \& Francis Group, 2015.

13. Burlington DB. Human factors and the FDA's goals: Improved medical device design. Biomed Instrum Technol 1996; 30(2): 107-109.

14. Lakbala P, Sobhani G, Lakbala M et al. Sharps injuries in the operating room. Environ Health Prev Med 2014; 19(5): 348-353.

15. Bharathan R, Aggarwal R, Darzi A. Operating room of the future. Best Pract Res Clin Obstet Gynaecol 2013; 27(3): 311-322.

16. Klein M, Andersen LPH, Alamili M et al. Psychological and physical stress in surgeons operating in a standard or modern operating room. Surg Laparosc Endosc Percutan Tech 2010; 20(4): 237-242.

17. Kenyon TAG, Urbach DR, Speer JB et al. Dedicated minimally invasive surgery suites increase operating room efficiency. Surg Endosc 2001; 15(10): 1140-1143.

18. Garbey M, Joerger $G$, Huang $A$ et al. An intelligent hospital operating room to improve patient health care. J Comput Surg 2015; 2(1): 3.

19. Tadeo JR. Improving operating room efficiency: a quality improvement project. Doctoral Thesis 2015; 130: 910-917.

20. Nundy S, Mukherjee A, Sexton JB et al. Impact of preoperative briefings on operating room delays. Arch Surg 2008; 143(11): 1068-1072.

21. Porta CR, Foster A, Causey MW et al. Operating room efficiency improvement after implementation of a postoperative team assessment. J Surg Res 2013; 180(1): 15-20.

22. Doll D, Wieferich K, Erhart T et al. Waiting for Godot. Eur J Anaesthesiol 2014; 31(7): 388-389.

23. Dexter F, Epstein RH, Marcon E et al. Estimating the incidence of prolonged turnover times and delays by time of day. Anesthesiology 2005; 102(6): 1242-1248.

24. Stahl J, Sandberg W, Daily B et al. Reorganizing patient care and workflow in the operating room: a costeffectiveness study. Surgery 2006; 139(6): 717-728.

25. Hanss R, Buttgereit B, Tonner PH et al. Overlapping induction of anesthesia: an analysis of benefits and costs. Anesthesiology 2005; 103(2): 391-400.

26. Torkki PM, Marjamaa RA, Torkki MI et al. Use of anesthesia induction rooms can increase the number of urgent orthopedic cases completed within 7 hours. Anesthesiology 2005; 103(2): 401-405.

27. Doll D, Kauf $P$, Wieferich $K$ et al. Implications of Perioperative Team Setups for Operating Room Management Decisions. Anesth Analg 2017; 124(1): 262-269.

28. Stepaniak PS, Vrijland WW, de Quelerij M et al. Working with a fixed operating room team on consecutive similar cases and the effect on case duration and turnover time. Arch Surg 2010; 145(12): 1165-1170.

29. Stepaniak PS, Heij C, Buise MP et al. Bariatric Surgery with Operating Room Teams that Stayed Fixed During the Day. Anesth Analg 2012; 115(6): 1384-1392.

30. Haynes AB, Weiser TG, Berry WR et al. A Surgical Safety Checklist to Reduce Morbidity and Mortality in a Global Population. N Engl J Med 2009; 360(5): 491-499.

31. Kopelman Y, Lanzafame RJ, Kopelman D. Trends in evolving technologies in the operating room of the future. JSLS 2013; 17(2): 171-173.

32. Health Care Financial Management Association (HCFMA). Achieving operating room efficiency through process integration, 2005.

33. Macario A, Vitez TS, Dunn B et al. Where are the costs in perioperative care? Analysis of hospital costs and charges for inpatient surgical care. Anesthesiology 1995; 83(6): 1138-1144.

34. Guerriero F, Guido R. Operational research in the management of the operating theatre: A survey. Health Care Manag Sci 2011; 14(1): 89-114.

35. Peters JA, Dean HM. Enhancing OR capacity and utilization. Health Financ Manage 2011; 65(1): 66-71.

36. Macario A. What does one minute of operating room time cost? J Clin Anesth 2010; 22(4): 233-236.

37. Shippert RD. A study of time-dependent operating room fees and how to save $\$ 100000$ by using timesaving products. Am J Cosmet Surg 2005; 22(1): 25-34.

38. Krupka DC, Sandberg WS. Operating room design and its impact on operating room economics. Curr Opin Anaesthesiol 2006; 19(2): 185-191. 